\title{
COMETARY NUCLEI
}

\author{
HANS RICKMAN \\ Astronomiska observatoriet, Box 515, S-75120 Uppsala, Sweden \\ E-mail HANS@ASTRO.UU.SE
}

\begin{abstract}
After a brief summary of current concepts on cometary nuclei, resulting from recent advances in observational techniques and laboratory experiments on cometary analogue materials, some of the outstanding problems facing theoretical modelling and interpretations of the new data is described. Emphasis is placed on the outgassing of species more volatile than water over the history of cometary nuclei, the structure of the nuclei in terms of porosity and tensile strength on a variety of different scales, and the volumetric importance and distribution of the refractory "dust" component. Near-surface interactions of dust grains and outflowing gas may be associated with the formation of large dust aggregates and mantling of the nuclei.
\end{abstract}

\section{Introduction}

Our picture of cometary nuclei has changed a great deal in recent years, in response to the stimulus of new, partially space-borne observations and laboratory experiments on simulated cometary materials. Nevertheless, the picture appears far from complete. While some aspects have become relatively well-established, others remain uncertain or controversial. This paper will discuss some of the issues which, in spite of recent progress, remain to be clarified. As a background to this discussion, let us summarize some current concepts about cometary nuclei, on which most authors agree.

- Cometary nuclei are icy bodies. The composition of the ice is dominated by $\mathrm{H}_{2} \mathrm{O}$, but other volatiles are retained as well even in comets that have come of age, like $\mathrm{P} / \mathrm{Halley}$. Examples are $\mathrm{CO}_{2}$ and $\mathrm{CO}$.

- The $\mathrm{H}_{2} \mathrm{O}$ ice is initially amorphous and thus able to trap the other volatiles at temperatures far above their standard sublimation levels. This, however, implies that the nuclei were formed and have been preserved at very low temperatures.

- As solar heating drives a crystallization front inward with release of trapped gases, considerable vapour pressures may build up, leading to fracture or splitting of the nuclei and/or to irregular bursts of outgassing.

- Compared with the ice and vapours, the "dust" component of the nuclear material is of equal or even greater importance by mass. It is composed of silicates and organic compounds in an apparently intricate mixture that may reflect presolar core-mantle grains.

- The structure of the nuclei is fluffy, implying bulk densities well below $1 \mathrm{~g} / \mathrm{cm}^{3}$ and a volumetrically important network of pores, perhaps from micron-scale up to macroscopic voids.

- The gas-dust interactions next to the surface involve a complicated scenario that leads to the entailment of grains with a wide size spectrum in the outflowing gas but also to the formation of "dust mantles" that reduce or quench the gas flow. 
- Due to the common occurrence of dust mantles, the outgassing from cometary nuclei is concentrated to "active regions" often referred to as local spots or vents. The nuclei evolve by opening up or closure of active spots, and a common end state may be complete mantling.

- The nuclear surface layers of new Oort cloud comets, or comets that have never before been close to the Sun, should bear witness of Gyrs of cosmic-ray exposure and so might have special optical and chemical properties.

- The size spectrum of cometary nuclei is very wide, extending upward to Chironsize objects or larger and downward to sub-km radii. Their spins are relatively slow and the shapes are irregular, often elongated.

These concepts, all consistent with Whipple's (1950) basic model, will be subject to critical scrutiny in the following sections, attempting to identify the weak points of our understanding and the pieces of information - both observational, experimental and theoretical - that are yet to be sought.

\section{Volatile outgassing}

Let us start by a statement that is hard to challenge : $\mathrm{H}_{2} \mathrm{O}$ is the dominant driver of cometary activity close to the Sun (heliocentric distance $r \lesssim 2-3 \mathrm{AU}$ ). This is most clearly borne out by the fact that nuclear production rates of other gases are always found to be at levels of several percent or less with respect to $\mathrm{H}_{2} \mathrm{O}$ (Mumma et al. 1993; Crovisier 1993). We can safely conclude that the volatile composition of cometary nuclei is $\mathrm{H}_{2} \mathrm{O}$-dominated. However, depending on the thermal properties and the detailed energetics of the surface layers, the $\mathrm{H}_{2} \mathrm{O}$ sublimation rate is bound to fall off rapidly with $r$, starting from within $5 \mathrm{AU}$, if solar heating is the only source of energy (Rickman 1992a). And yet, one often observes grain comae or tails at $r>5 \mathrm{AU}$ despite the fact that $\mathrm{H}_{2} \mathrm{O}$ sublimation cannot be expected to lift the grains off the nuclei. Reasons to conclude on other outgassing mechanisms in distant comets have been reviewed by Meech (1993), the basic argument being that large quantities of grains are driven out from the nuclei at distances $(r \gtrsim 10$ AU) where water ice has to remain inert. Fig. 1, from Meech (1993), shows several examples of long-period (LP) comets exhibiting a continuous decline of the activity level with increasing $r$, and comet $\mathrm{P} / \mathrm{Halley}$ is seen to have returned, temporarily, to the typical LP comet activity level during its recent outburst.

The driver of the distant activity, unless an extremely strong internal heat source is invoked, has to be the outgassing of species more volatile than $\mathrm{H}_{2} \mathrm{O}$. Moreover, since these are just minor constituents, we are led to the concepts of subsurface production and diffusion of vapours to the surface through the porous icy matrix of the nuclei. A priori, one may then consider two different alternatives :

- individual ices sublimating independently at different depths; or

- release of gases, trapped in amorphous $\mathrm{H}_{2} \mathrm{O}$ ice (Bar-Nun et al. 1987), as the ice crystallizes.

The first of these scenarios requires us to explain how volatile ices, e.g. CO, can be retained in the $\mathrm{P} / \mathrm{Halley}$ nucleus, and the second raises the question of survival of amorphous ice. The problem of retention of $\mathrm{CO}$ ice involves two main 

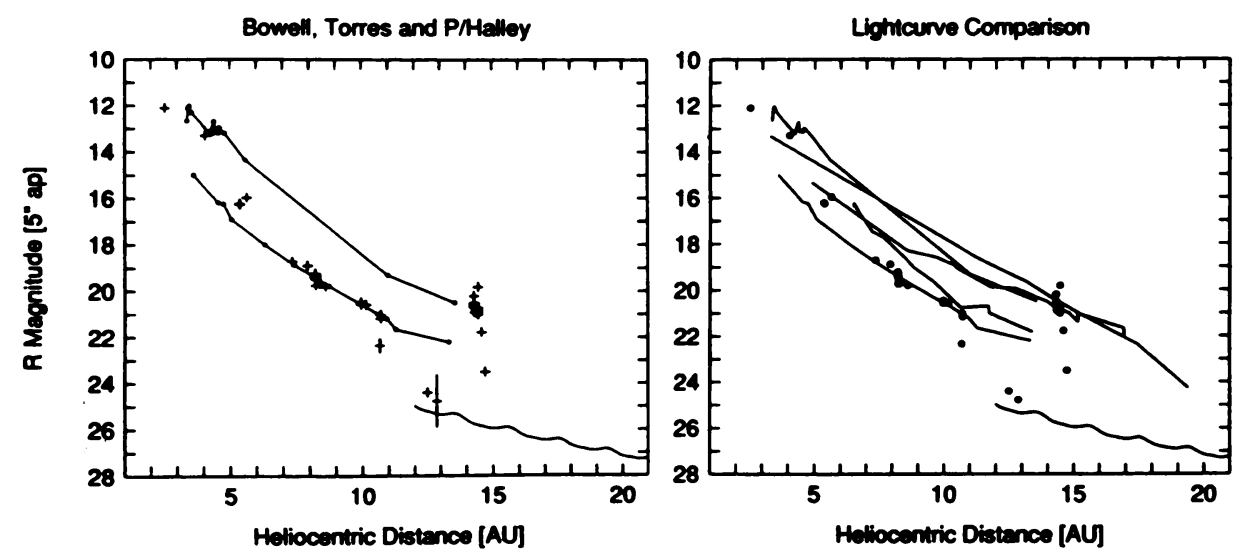

Fig. 1. Left : Light curves of comets 1982 I Bowell (upper curve), $1987 \mathrm{~V}$ Torres (lower curve) and 1986 III $\mathrm{P} /$ Halley (crosses); the curve to the lower right is $\mathrm{P} / \mathrm{Halley}$ 's theoretical bare nucleus light curve. Right: Light curve comparison for $\mathrm{P} / \mathrm{Halley}$ (filled circles and lower-right curve) and five LP comets (solid curves). ¿From Meech (1993).

issues. First, the radiogenic heating due to the decay of ${ }^{40} \mathrm{~K},{ }^{232} \mathrm{Th},{ }^{235} \mathrm{U}$ and ${ }^{238} \mathrm{U}$ included into the silicate fraction of the dust component causes a rise of the central temperature $\left(T_{c}\right)$ which, depending on the size and thermal diffusivity of the nucleus, may reach the level $\left(T_{c} \sim 30 \mathrm{~K}\right)$ where $\mathrm{CO}$ ice sublimates rapidly. This would lead to an outward flow of $\mathrm{CO}$ vapour and recondensation of the ice closer to the surface (Whipple and Stefanik 1966), and consequently, upon capture into a short-period (SP) orbit, the $\mathrm{CO}$ ice might be concentrated in the outer layers of the nucleus which are the first to be eroded away. Thus the second issue is whether any $\mathrm{CO}$ ice remains after a decrease to, say, half the initial radius following surface insolation and $\mathrm{H}_{2} \mathrm{O}$ ice sublimation. Model calculations are needed in order to solve this problem.

It seems fair to say that the choice between the two above scenarios, and the preference for the second one among most authors, is largely governed by considerations of how and where cometary nuclei were formed. The ice in molecular clouds is known to be amorphous (see Greenberg 1989a) and extrapolation of laboratory data (Schmitt et al. 1989) indicates that the crystallization time scale exceeds the age of the Solar System at $T \lesssim 75 \mathrm{~K}$. Thus the assumption that cometary nuclei formed out of primordial grains of the presolar cloud leads directly to the concept of amorphous ice. Even if the grains were thermally processed, e.g. upon entry into the solar nebula (Lunine et al. 1991), recondensation of ice at $T \lesssim 50 \mathrm{~K}$ would still form the amorphous phase (see, however, Shul'man 1992 for a different point of 
view). The problem then reduces to that of the thermal history of the nucleus and the survival of amorphous ice in the presence of radiogenic and solar-induced heating. One important difference between this problem and that of $\mathrm{CO}$ ice retention is that crystallization, in contrast to $\mathrm{CO}$ ice sublimation, is an exothermic process.

Recently models were computed by Haruyama et al. (1993) for the thermal evolution of cometary nuclei over the age of the Solar System in response to radiogenic heating by the above-mentioned isotopes. The nuclei were assumed to consist of a mixture of amorphous ice and dust in the form of core-mantle grains (Greenberg $1989 \mathrm{~b})$. For the thermal conductivity of amorphous ice, the experimentally determined value by Kouchi et al. (1992) was used, which is roughly a factor $10^{4}$ lower than earlier, theoretical estimates (e.g. Klinger 1980). This has an important consequence in facilitating runaway crystallization, caused by the latent heat release of $90 \mathrm{~J} / \mathrm{g}$, since the heat becomes essentially locked up in sizeable nuclei for the age of the Solar System. Indeed, as an example, it was found that for an optimal (though realistic) dust/ice ratio of unity and an ad hoc (though realistic) assumption that the porous grain matrix has a bulk conductivity 100 times less than the individual grains, all nuclei larger than $2 \mathrm{~km}$ radius would undergo runaway crystallization.

This is an interesting indication, highlighting a potential problem in explaining the preservation of amorphous ice over the age of the Solar System. More realistic models should now be devised, incorporating gas release and recondensation and exploring a range of values for the amorphous ice conductivity as well as for the silicates/organics ratio of the dust component, assuming chondritic abundances of radioisotopes only for the silicate fraction.

For dynamically old comets, especially short-period ones, an additional problem arises in clarifying what happens in response to solar heating, assuming the amorphous ice indeed survives until the entry into SP orbits. Early models adapted to the case of $\mathrm{P} / \mathrm{Halley}$ (Prialnik and Bar-Nun 1987) indicated a series of major crystallization bursts that in due time would reach the central region of the nucleus. The amplitude of the events proved to be a function of the model parameters chosen (Prialnik and Bar-Nun 1988), but a progressive crystallization of the whole nucleus remains a likely outcome. Furthermore, it has been suggested (e.g. Klinger 1983 ) that, for typical Jupiter family comets (perihelion distance $q \simeq 1.5 \mathrm{AU}$; orbital period $P \simeq 7 \mathrm{yr}$ ), orbital mean temperatures in excess of the critical range where fast crystallization ensues should imply a rapid phase transition of the whole nucleus into the crystalline state. The models by Espinasse et al. (1989; 1991), tailored to the case of $\mathrm{P} /$ Churyumov-Gerasimenko and utilizing a complete set of equations for the coupled gas and heat diffusions in a porous nucleus with a propagating crystallization front associated with gas release, indeed indicated runaway behaviour by extrapolation beyond the 35 years spanned by the calculations.

However, similar models were recently developed by Tancredi et al. (1993), using a different numerical approach and exploring more realistic values of physical and chemical parameters over a longer time span. As a result, treating a nucleus with equal amounts of dust and ice and with $10 \%$ trapped $\mathrm{CO}$ in the amorphous $\mathrm{H}_{2} \mathrm{O}$ ice, the crystallization was found to start out very rapidly in agreement with Espinasse et al., but it gradually slows down so that the speed of the front approaches that of surface erosion. Consequently, in this case extrapolation beyond 500 years indicates 
a time scale of $\sim 10^{4}$ years for complete crystallization (Fig. 2). This is of the same order as the dynamical lifetime of observable Jupiter family comets. Instrumental for the reduction of the crystallization rate is the absorption of part of the latent heat of crystallization by the inert dust component as well as the additional expense of heat for sublimating the $\mathrm{CO}$ ice that condenses below the front.
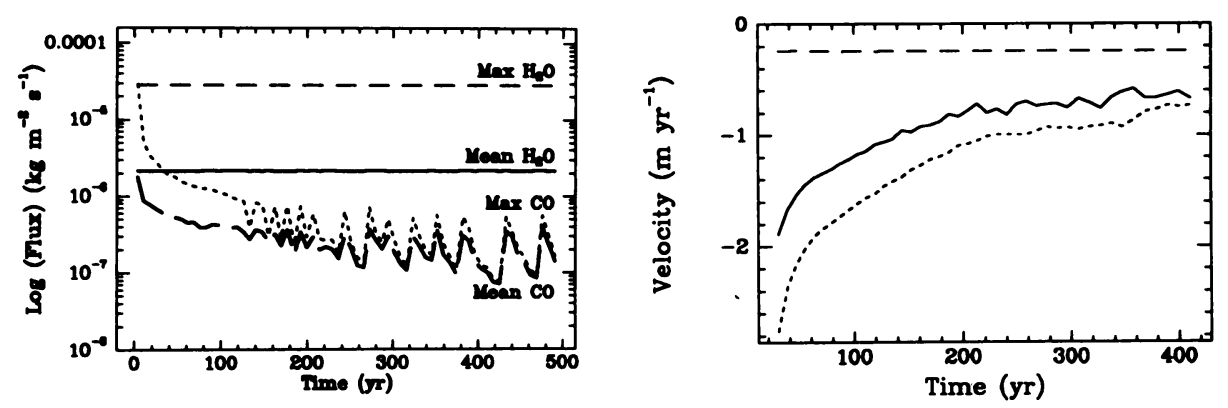

Fig. 2. Left : $\mathrm{H}_{2} \mathrm{O}$ and $\mathrm{CO}$ production rates for a model nucleus with $10 \%$ trapped $\mathrm{CO}$ moving in a Jupiter family orbit; thin curves indicate the maximum rates (at perihelion for $\mathrm{H}_{2} \mathrm{O}$ ) and thick curves indicate the mean values over one orbital revolution. Right : Surface erosional velocity (dashed curve), velocity of the crystallization front (solid curve), and velocity of the recondensed $\mathrm{CO}$ ice zone (dotted curve) for the same model. From Tancredi et al. (1993).

A common feature of most models so far computed is that crystallization proceeds in bursts. Such bursts may apparently occur over a wide range of heliocentric distance, and they have thus been invoked as a tentative explanation for the $\mathrm{P} /$ Halley outburst at $r \simeq 14 \mathrm{AU}$ (Prialnik and Bar-Nun 1993; Schmitt et al. 1993). Moreover, for the case of trapped $\mathrm{CO}$, they cause large variations of the $\mathrm{CO}$ production rate between successive apparitions. For $q \simeq 1.5 \mathrm{AU}$ this is always several orders of magnitude below the peak $\mathrm{H}_{2} \mathrm{O}$ production rate of an unmantled, icy nucleus. Interestingly, though, the situation appears quite different for comets such as $\mathrm{P} /$ Gehrels 3 or $\mathrm{P} /$ Smirnova-Chernykh with $q \simeq 3.5 \mathrm{AU}$. Even for this kind of orbit crystallization is quite fast, at least initially (Tancredi et al. 1993), and one would expect that the activity of large- $q$ Jupiter family comets might be largely CO-driven - an indication that remains to verify observationally.

\section{Structural properties}

An important observational constraint on the porosity of cometary nuclei comes from mass determination via the nongravitational effects in the orbital motions. However, some of the relevant parameters cannot be observed and must hence be estimated on the basis of physical modelling. The determination and analytical 
representation of nongravitational effects in orbital solutions is reviewed by Yeomans (this volume). For the purpose of mass determination, use is made only of the principal effect, i.e., the perturbation of the mean motion $(n=2 \pi / P)$ or orbital period $(P)$. Integrated over one orbital revolution this amounts to :

$$
\Delta P=\frac{a^{5 / 2}}{\left(G M_{\odot}\right)^{3 / 2}} \cdot 6 \pi m\left\langle u_{g}\right\rangle \cdot \frac{Q_{m}}{M}\left\{\left\langle C_{r}\right\rangle E+\left\langle C_{t}\right\rangle T\right\}
$$

where $a$ is the orbital semimajor axis, $G$ is the gravitational constant, $M_{\odot}$ and $M$ are the masses of the Sun and the comet, respectively, $m$ is the mean mass of the gas molecules flowing out from the nucleus, $\left\langle u_{g}\right\rangle$ is an orbital average of the mean outflow velocity, and $Q_{m}$ is the maximum gas production rate from the cometary nucleus. The curly bracket features the orbital averages of the direction cosines $\left(C_{r}\right.$ and $C_{t}$ ) of the force vector along the radial and transverse directions in the orbital plane, and $E$ and $T$ are parameters characterizing the gas production curve $Q(t)$ (Rickman 1992b). $E$ measures the perihelion asymmetry of the gas production curve and $T$ measures its peakedness; it should be noted that $\left\langle C_{r}\right\rangle$ may not be meaningful unless $C_{r}$ is nearly constant.

Solving for $M$ from Eq. (1) requires knowledge of the gas production curve and the velocity vector of the average gaseous outflow. The incompleteness of such knowledge translates into error bars on the derived value of $M$. Considering the velocity modulus factor, $\left\langle u_{g}\right\rangle$, its value depends on the geometry of the outflow from the nucleus, and modelling of this involves 2-D hydrodynamical simulations for an expanding gas-dust mixture (Kitamura 1986). Special issues that have been discussed include the averaging of the vertical velocity component in the Knudsen layer next to the nucleus (Bisikalo et al. 1989), the return flux due to molecular collisions and partial thermalization of the velocity distribution (Crifo 1987), and the additional recoil momentum of gas molecules returning to the surface after accelerating the dust grains (Peale 1990). The result is a velocity vector perpendicular to the local nuclear surface, whose magnitude can be written : $u_{\ell}=\zeta_{\ell} v_{t h}\left(T_{s}\right)$ where $v_{t h}=\sqrt{8 k T_{s} / \pi m}$ is the thermal speed of the gas molecules and $\zeta_{\ell}$ is a local momentum transfer coefficient. Until now the values used for $\zeta_{\ell}$ by different authors (Sagdeev et al. 1988; Rickman 1989; Peale 1990) have been crude estimates based on simplified arguments, and realistic model calculations remain to be done.

The mean outflow velocity $\mathbf{u}_{g}$ is a global average of the local velocity vector $\mathbf{u}_{\ell}$ over the whole gas-producing area during the complete spin of the nucleus, weighted by the local gas flux. Its modulus is $u_{g}$ and its direction cosines are $-C_{r}$ and $-C_{t}$. The vectorial averaging implies that $u_{g}$ should be smaller than the global average of $u_{\ell}$, so we can formally write : $u_{g}=\zeta_{g} u_{\ell}$, introducing $\zeta_{g}<1$ as a global momentum transfer coefficient. We are currently faced with different models for the nuclear spin of comets as well as the activity distribution around their nuclear surfaces, influencing the estimates of $\zeta_{g}, C_{r}$ and $C_{t}$ (see Fig. 3). On the one hand, the spin is sometimes modelled as pure rotation around a fixed axis or one precessing due to the torque exerted by asymmetric outgassing from a non-spherical nucleus (e.g. Whipple and Sekanina 1979; Sekanina 1984; Sekanina 1992) and sometimes as a complex motion involving rotation, precession and nutation excited by the 
jet torque (Sekanina 1987; Rickman 1989; Banaszkiewicz and Szutowicz 1990). On the other hand, there is also an unsettled issue about the relative contributions to cometary outgassing from isolated jets emerging from local active spots and from broad background fans with roots seated all around the nuclei. In P/Halley the jets appear to dominate (Keller 1990), but it appears premature to extrapolate this result to all comets. Concerning the spin model, we also have good evidence for a complex state of rotation for P/Halley (Belton 1991), and it would seem natural to apply such models in general.
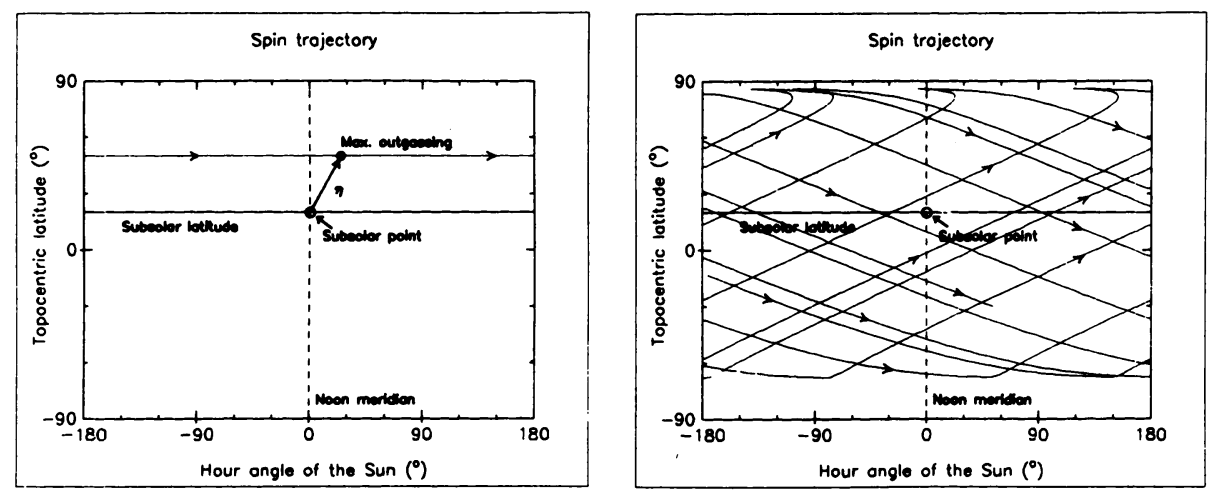

Fig. 3. Left: Map of a cometary nucleus with a fixed spin axis; an active spot describes the horizontal trajectory with arrows indicating the direction of rotation, and a thermal lag causes the point of maximum outgassing to be displaced from the subsolar meridian. Right: Corresponding trajectory during one month for an active spot on a spheroidal nucleus rotating around the long body axis, which in turn precesses around a fixed angular momentum axis. The maximum outgassing in this case tends to occur closer to the subsolar latitude. From Rickman (1989).

However, Eq. (1) shows that the interpretation of the nongravitational effect goes hand in hand with that of the perihelion asymmetry of the gas production curve, i.e., the light curve asymmetry. Recent work (Yeomans and Chodas 1989; Festou et al. 1990; Sekanina 1992) has convincingly demonstrated the validity of Bessel's (1836) conjecture about the importance of the 'radial' term in this equation. As yet, the jet outflow model with pure rotation offers the only straightforward explanation to the frequent occurrence of strong light curve asymmetries with both preperihelion and postperihelion peaks among short-period comets by means of the seasonal variation of insolation onto the dominant active spot. For comet $\mathrm{P} / \mathrm{Halley}$ the coexistence of an important postperihelion outgassing excess and a rotational motion involving significant wobbling remains unexplained, and the constancy of the nongravitational effect over 30 orbital revolutions appears enigmatic. 
The lack of knowledge about the distribution of outgassing activity around the nuclear surface and the rotational state for any given comet naturally transforms into an error bar on $\zeta_{g}$, but unless extreme cases are considered, this one is unimportant compared to that on $\zeta_{\ell}$. Of much greater consequence is the fact that one-spot models with pure rotation (Sekanina 1992) imply a general correlation between $E$ and $\left\langle C_{t}\right\rangle$ so that the radial and transverse terms in Eq. (1) tend to act in concert and to contribute comparably to the total effect. More complex models would generally attach less significance to the transverse term (Rickman 1989); and even a small, though unknown, thermal lag may upset the above result for comets with nearly symmetric gas production curves. To summarize the current situation, nuclear masses may be estimated for comets with large values of $|E|$ from Eq. (1) using a model calculation of $\left\langle u_{g}\right\rangle$ and observational data on $Q_{m}$ and $E$, taking as an ad hoc estimate : $\left\langle C_{r}\right\rangle E+\left\langle C_{t}\right\rangle T \approx E$. As a consequence, we are limited to comets with well-observed, asymmetric gas production curves for which $Q_{m}$ and $E$ are known with decent accuracy. Even so, an unavoidable error of a factor $2-3$ comes from the uncertainties of the above-described estimates of $\left\langle u_{g}\right\rangle$ and $\left\langle C_{r}\right\rangle E+\left\langle C_{t}\right\rangle T$. Only in exceptional cases, such as that of $\mathrm{P} / \mathrm{Halley}$, can this modelling uncertainty be reduced. However, better knowledge of the gas production curves would lead to some improvement, e.g. when considering the typical mean density of a statistical sample of cometary nuclei. From the cases analyzed as yet (Rickman 1989; Rickman et al. 1992), it appears that cometary nuclei are very porous (mean density $\rho \lesssim 0.5$ $\left.\mathrm{g} / \mathrm{cm}^{3}\right)$.

Porosity means a fluffy structure, but do we know over which scales the fluffiness extends? Donn (1990) advocates a fractal build-up of cometary nuclei resulting from formation by low-velocity random accretion. Numerical simulations (Daniels and Hughes 1981) yield open structures with a fractal dimension $D \approx 2$, as illustrated by Fig. 4a from Hughes (1988), i.e., a density decreasing with linear size $(R)$ as $\rho \propto R^{-1}$. This obviously cannot hold over many orders of magnitude in real nuclei, whose bulk packing factors appear to be $\sim 0.1$ judging from the above density estimate. Hence a great deal of compaction must have occurred during the accretion process (Donn 1990). Information on the formation conditions is hence stored in the inhomogeneity of the nuclei, in particular the average variation of the packing factor with the volume of the sample considered. Related to this is the tensile strength spectrum, i.e. the variation of the typical tensile strength of the material with the sample area considered, since this may be affected by the collisional merging of particles and dependent on the velocities involved.

In addition to the apparently fluffy structure, some evidence for the random low-velocity accretion scenario is present in the nuclear photometric observations indicating generally elongated shapes (Jewitt and Meech 1988), as expected from numerical simulations (Fig. 4b), and perhaps also in the waist-like feature on the P/Halley nucleus (Keller 1990) that appears to mark the intersection of two large cometesimals. But affirmative evidence for a fractal-like build-up, and characterization of the associated porosity and tensile strength spectra, are very difficult to realize and offer a great challenge for future cometary exploration. Near-surface in situ studies by landers and penetrators may be insufficient as long as they sample only the transformed, crystalline crust (Sect. 2) where sintering may have modi- 

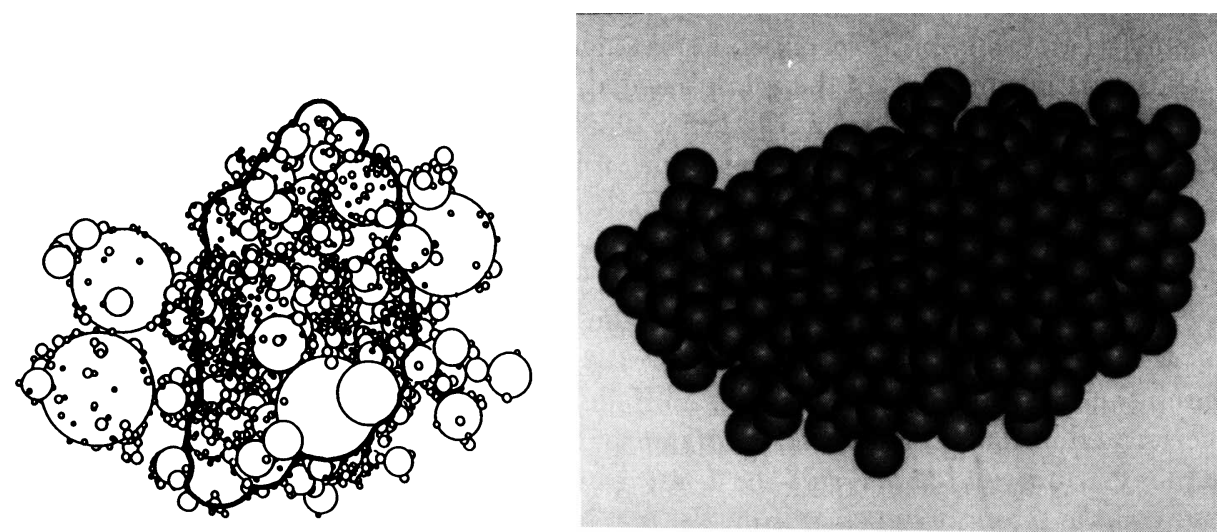

Fig. 4. Left : Fractal model of a newly accreted cometary nucleus, self-similar from micrometric to kilometric scales; the thick contour indicates the shape after considerable surface erosion has occurred. After Hughes (1988). Right: Simulated cometary nucleus formed by 1000 randomly accreted spheres of equal size. From Jewitt and Meech (1988).

fied the microstructure (Kochan et al. 1989). Recently modifications of the pore structure by the pressure of released gases at the crystallization front were given a preliminary treatment (Prialnik et al. 1993), indicating the possibility of catastrophic tensile failure that would probably cause fragments to be split off the nucleus.

Analysis of cometary splittings (Sekanina 1982) offers an interesting possibility to probe the tensile strength spectrum over a wide range, if supplemented by further data on cometary dust trails or meteor streams and radar or microwave observations of the near-nucleus regions of comets of different degrees of activity. Comet 1993e $\mathrm{P} /$ Shoemaker-Levy 9 is a particularly fascinating case, having split up as it passed within Jupiter's Roche limit in July 1992 to an as yet unknown miss distance (Carusi et al. 1993). On this occasion tensile failure occurred at least on a large scale, yielding the $\sim 20$ large fragments now seen as separate comets. If some pieces should just barely escape collision with Jupiter in July 1994, their splitting behaviour should be monitored as closely as possible.

\section{The dust component}

How much refractories do cometary nuclei contain in relation to the volatiles? We are interested in finding the value, or the range of values, of the dust/ice ratio. This is not amenable to direct observations, but one often considers dust/gas ratios in cometary comae based on ratios of continuum brightness to band emissions as indicators. However, it is important to note that there is a long way to go from this indicator to the desired quantity. The scattered light from the dust coma is dominated by a relatively narrow range of grain sizes, which apparently does not 
contribute a great deal to the total mass. It is well known that comets vary a lot in appearance and that some appear dusty, while others appear gassy, but one cannot infer with certainty a difference in nuclear composition from this.

In situ measurements of the grain size distribution are available for the $\mathrm{P} / \mathrm{Halley}$ coma from the S/C encounters in March 1986. Fig. 5, from Curdt and Keller (1989), summarizes these data. The grains with radii $a \sim 1 \mu \mathrm{m}$ that dominate the visual scattered light show a relatively rapid fall-off (cumulative power-law mass index $\alpha \simeq 0.9$ ) toward larger sizes, as measured by the PIA and DID dust detectors, and by simple extrapolation one would find a rather small dust/gas production ratio $\sim 0.2$, close to the pre-encounter expectation from analyses of dust comae and tails in other comets (Divine et al. 1986). But accounting for the large particles that shook the Giotto spacecraft (Curdt and Keller 1989) and the total dust fluence that decelerated it (Edenhofer et al. 1987), one finds a much flatter distribution $(\alpha \simeq 0.2)$ at $a \gtrsim 0.1 \mathrm{~mm}$, and as a result the dust/gas ratio is considerably increased. In fact the result is strongly dependent on the maximum grain size involved. This general property seems confirmed by the GEM-DIDSY detections at P/Grigg-Skjellerup (McDonnell et al. 1993) and the radio science indication of an even larger impacting particle (Pätzold et al. 1993).

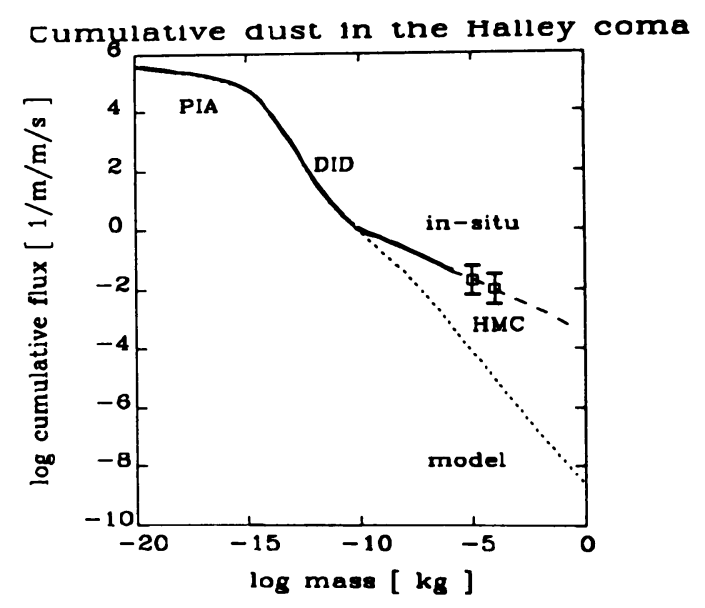

Fig. 5. Cumulative flux of dust grains of mass $>m$ registered in the $\mathrm{P} / \mathrm{Halley}$ coma by PIA and DID during the last two minutes before closest approach, depicted by the solid curve. The two data points to the right are derived from $S / C$ attitude changes measured by the HMC camera. The dotted line shows the pre-encounter model distribution. From Curdt and Keller (1989).

A total dust/gas production ratio of order unity is indicated, but the exact value cannot yet be inferred. Nonetheless, this is an important matter, since on cosmochemical grounds the presolar grains should have a dust/ice ratio close to 1 (Greenberg 1989b), and the finding of a value far from this would indicate a severe problem for the current understanding of comet formation. Values of $2-3$ for the 
dust/gas production ratio correspond to a cutoff at the "maximum liftable size" (mass $\sim 1 \mathrm{~kg}$; Keller 1989), but even though the existence of such large particles in the P/Halley coma is indicated by radar observations (Campbell et al. 1989), the inference is still risky since the meaning of the concept is not clear (see below). Furthermore, there may be large variations of the amount of dust produced as large grains during the course of the apparition, as temporary dust mantles are formed and blown away, so it appears premature to conclude that cometary nuclei contain substantially more dust than ice. Nevertheless, the issue is of great importance for several reasons. Not only would a dust/ice ratio of $2-3$ cast doubt on the reference picture of comet formation, but it would call upon a new picture of cometary material as a refractory matrix with icy inclusions - an "icy dirt ball" (Keller 1989). This might have important consequences for the understanding of dust mantle development (Kührt and Keller 1993).

Of considerable interest is also the ratio of organics to silicates in the dust component. The total production rate of complex, carbon-bearing molecules cannot yet be determined by remote-sensing techniques, even though it is probably related to the strength of the emission near $3.4 \mu \mathrm{m}$ (e.g. Baas et al. 1986), and though such molecules may provide a source for the most conspicuous radicals observed in visual spectra as indicated by the CN jets (A'Hearn et al. 1986) or $\mathrm{C}_{2}$ jets (Cosmovici et al. 1988) in $\mathrm{P} /$ Halley. We are thus limited to the snapshot picture of relative CHON grain abundance offered by the PIA instrument during the Giotto flyby (Langevin et al. 1987), indicating a production rate of organics comparable to that of silicates.

A very important but still elusive property of cometary material is the size distribution of dust grains residing inside the nuclei. There is an apparent conflict between the presence of coma grains with a very wide size spectrum and the reference picture of cometary formation, where the starting point is a unimodal distribution of presolar grains of submicron size with refractory cores and icy mantles. The most natural solution may be the agglomeration of these submicron refractory cores into larger structures in the near-surface layers as a consequence of the outgassing process. Laboratory sublimation experiments, like KOSI (Grün et al. 1991), have provided a wealth of useful information relevant to the understanding of such processes, but theoretical modelling is lagging behind and thus it remains difficult to predict the outcome in real comets exposed to varying insolation conditions as they move around their orbits.

In previous modelling work it has generally been assumed that the whole observed size spectrum of dust grains resides "ready-made" in the nuclear ice, whereupon one applies concepts like the critical radius $a_{c}$ of the maximum liftable grains for the outgassing flux $Z$, initially introduced by Whipple (1950) :

$$
a_{c}=\frac{C_{D} u_{\ell} m Z}{\frac{8}{3} \rho_{g} R\left(\frac{4}{3} \pi G \rho-\Omega^{2} \cos ^{2} \beta\right)}
$$

where $C_{D}$ is the drag coefficient, $\rho_{g}$ is the density of the grain, $R$ is the radius of the nucleus, $\Omega$ is its spin angular velocity and $\beta$ is the latitude (Rickman et al. 
1990). Using this, one models the accumulation of grains with $a>a_{c}$ on a freely outgassing surface until a critical level of coverage is reached (e.g. Shul'man 1972) such that a dust mantle is formed. A physical theory for this dust mantle (e.g. Shul'man 1981; Horányi et al. 1984; Rickman and Fernández 1986) is then used to follow its evolution and to check whether it remains stable against the gas pressure building up by ice sublimation underneath, or whether mantle blowoff occurs.

There are several problems plaguing this kind of models. First, their predictive power is weak since many parameters are badly constrained and a wide range of outcomes remains possible. For instance, the build-up of a dust mantle appears very slow according to the results of Fanale and Salvail (1984), whereas Rickman et al. (1990) found mantles to be formed and destroyed on very short time scales. Secondly, as noted above, there is an inconsistency in the scenario used, since the problem of forming the large dust aggregates with $a>a_{c}$ is shortcut. A step toward a treatment of this problem was taken in the framework of the "friable sponge" model (see Horányi and Kecskeméty 1983) using percolation theory to predict the grain size distribution. A further-reaching idea that remains to be explored is to model the gas-grain interactions in the boundary layer where the $\mathrm{H}_{2} \mathrm{O}$ vapour feeding the outflow is produced (Rickman 1992a). In this layer there should be a gradual stripping of the icy mantles off the grains and a vigorous gas flow causing the grains to move relative to each other and accrete into larger structures but also tending to tear these structures apart.

It seems unlikely to have $\mathrm{mm}$ - or cm-sized aggregates formed in this way though their existence is indisputable based on observations of cometary fireballs or dust trails. An attractive picture is that these form as a result of mantle blowoff, thus requiring to have unstable mantles of rather frequent occurrence. Thus the large grains involved in the above estimate of the total dust/gas production ratio may be expelled from the nucleus in a peculiar manner, which casts doubt on the application of the critical radius as given in Eq. (2). To improve our understanding of the evolution of cometary nuclei as well as their composition and the origin of the meteoroidal and zodiacal dust complex, it is an urgent task to develop models for the physics of the near-surface layers of the nuclei, and to present a self-consistent picture of the formation of large dust aggregates and mantles. To this end the experience recently gained through laboratory experiments should be exploited, but we may need even more input from such work, e.g. concerning the cohesive strength of dust aggregates.

\section{Conclusion}

The reference picture of cometary nuclei sketched in the introduction represents a real achievement, when compared with what was usually thought before the age of space exploration of comets, but it is not at all finished -- in fact, the picture is just beginning to emerge. There are still many tasks that have to be better fulfilled and matters that one should learn much more about. Needless to say, nothing appears basically wrong about the reference picture, but most aspects remain quite uncertain. Thus our knowledge of the true nature of cometary nuclei is still very limited, and the picture can only be accepted or discarded in favour 
of something better after further advances have been made. This is a matter of both observations, experiments and theoretical models, and it may be a necessary step to have future cometary space missions such as those now under study, e.g. Rosetta.

\section{References}

A'Hearn, M.F., Hoban, S., Birch, P.V., Bowers, C., Martin, R. and Klinglesmith III, D.A. : 1986, "Cyanogen jets in comet Halley". Nature, 324, 649-651.

Baas, F., Geballe, T.R. and Walther, D.M. : 1986, "Spectroscopy of the 3.4 micron emission feature in comet Halley". Astrophys. J. Lett., 311, L97-L101.

Banaszkiewicz, M. and Szutowicz, S. : 1990, "Thermal Modelling of Precessing Cometary Nuclei". In Asteroids, Comets, Meteors III (C.-I. Lagerkvist, H. Rickman, B.A. Lindblad, M. Lindgren, Eds.), 239-242, Uppsala Univ. Reprocentralen.

Bar-Nun, A., Dror, J., Kochavi, E. and Laufer, D. : 1987, "Amorphous water ice and its ability to trap gases". Phys. Rev., B 35, 2427-2435.

Belton, M.J.S. : 1991, "Characterization of the Rotation of Cometary Nuclei". In Comets in the Post-Halley Era, vol. 2 (R.L. Newburn, Jr., M. Neugebauer, J. Rahe, Eds.), 691721, Kluwer, Dordrecht.

Bessel, F.W. : 1836, "Bemerkungen über mögliche Unzulänglichkeit der die Anziehungen allein berücksichtigenden Theorie der Kometen". Astron. Nachr., 13, 345-350.

Bisikalo, D.V., Marov, M.Ya., Shematovich, V.I. and Strel'nitskij : 1989, "The flow of the subliming gas in the near-nuclear (Knudsen) layer of the cometary coma". Adv. Space Res., 9, (3)53-(3)58.

Campbell, D.B., Harmon, J.K. and Shapiro, I.I. : 1989, "Radar observations of comet Halley". Astrophys. J., 338, 1094-1105.

Carusi, A., Marsden, B.G. and Valsecchi, G.B. : 1993, "The probable collision of P/Shoemaker-Levy 9 (1993e) with Jupiter in 1994". Planet. Space Sci., in press.

Cosmovici, C.B., Schwarz, G., Ip, W.-H. and Mack, P. : 1988, "Gas and dust jets in the inner coma of comet Halley". Nature, 332, 705-709.

Crifo, J.F. : 1987, "Improved gas-kinetic treatment of cometary water sublimation and recondensation : application to comet P/Halley". Astron. Astrophys., 187, 438-450.

Crovisier, J. : 1993, "Molecular abundances in comets". Planet. Space Sci., in press.

Curdt, W. and Keller, H.U. : 1989, "Large Dust Particles along the Giotto Trajectory". MPAE Report, MPAE-W-55-89-19.

Daniels, P.A. and Hughes, D.W. : 1981, "The Accretion of Cosmic Dust - A Computer Simulation". Mon. Not. R. Astr. Soc., 195, 1001-1009.

Divine, N., Fechtig, H., Gombosi, T.I., Hanner, M.S., Keller, H.U., Larson, S.M., Mendis, D.A., Newburn Jr., R.L., Reinhard, R., Sekanina, Z. and Yeomans, D.K. : 1986, "The Comet Halley dust and gas environment". Space Sci. Rev., 43, 1-104.

Donn, B.D. : 1990, "The formation and structure of fluffy cometary nuclei from random accumulation of grains". Astron. Astrophys., 235, 441-446.

Edenhofer, P., Bird, M.K., Brenkle, J.P., Buschert, H., Kursinski, E.R., Mottinger, N.A., Porsche, H., Stelzried, C.T. and Volland, H. : 1987, "Dust distribution of comet P/Halley's inner coma determined from the Giotto Radio-Science Experiment". Astron. Astrophys., 187, 712-718.

Espinasse, S., Klinger, J., Ritz, C. and Schmitt, B. : 1989, "A method of estimating temperature profiles and chemical differentiation in the near surface layers of porous comet nuclei - first results for comet $\mathrm{P} / \mathrm{Churyumov-Gerasimenko".} \mathrm{In} \mathrm{Physics} \mathrm{and}$ Mechanics of Cometary Materials, ESA SP-302 (J. Hunt, T.D. Guyenne, Eds.), 185190, ESA Publ. Div.

Espinasse, S., Klinger, J., Ritz, C. and Schmitt, B. : 1991, "Modeling of the Thermal Behavior and of the Chemical Differentiation of Cometary Nuclei". Icarus, 92, 350-365. 
Fanale, F.P. and Salvail, J.R. : 1984, "An idealized short-period comet model : Surface insolation, $\mathrm{H}_{2} \mathrm{O}$ flux, dust flux, and mantle evolution". Icarus, 60, 476-511.

Festou, M.C., Rickman, H. and Kamél, L. : 1990, "Using comet light-curve asymmetries to predict comet returns". Nature, 345, 235-238.

Greenberg, J.M. : 1989a, "Interstellar Dust : an Overview of Physical and Chemical Evolution". In Evolution of Interstellar Dust and Related Topics (A. Bonetti, J.M. Greenberg, S. Aiello, Eds.), 7-52, North Holland.

Greenberg, J.M. : 1989b, "From interstellar dust to comet dust and interplanetary matter". In Highl. of Astron. (D. McNally, Ed.), 8, 241-250, IAU.

Grün, E., Bar-Nun, A., Benkhoff, J., Bischoff, A., Düren, H., Hellmann, H., Hesselbarth, P., Hsiung, P., Keller, H.U., Klinger, J., Knölker, J., Kochan, H., Kohl, H., Kölzer, G., Krankowsky, D., Lämmerzahl, P., Mauersberger, K., Neukum, G., Oehler, A., Ratke, L., Roessler, K., Schwehm, G., Spohn, T., Stöffler, D. and Thiel, K. : 1991, "Laboratory simulation of cometary processes : Results from first KOSI experiments". In Comets in the Post-Halley Era, vol. 1 (R.L. Newburn, Jr., M. Neugebauer, J. Rahe, Eds.), 277-297, Kluwer, Dord recht.

Haruyama, J., Yamamoto, T., Mizutani, H. and Greenberg, J.M. : 1993, "Thermal History of Comets during Residence in the Oort Cloud : Effect of Radiogenic Heating in Combination with the Very Low Thermal Conductivity of Amorphous Ice”. J. Geophys. Res. Planets, in press.

Horányi, M. and Kecskeméty, K. : 1983, "Cometary dust". In Asteroids, Comets, Meteors (C.-I. Lagerkvist, H. Rickman, Eds.), 269-272, Uppsala Univ. Reprocentralen.

Horányi, M., Gombosi, T.I., Cravens, T.E., Körösmezey, A., Kecskeméty, K., Nagy, A.F. and Szegö, K. : 1984, "The friable sponge model of a cometary nucleus". Astrophys. $J ., 278,449-455$.

Hughes, D.W.: 1988, "Cometary Magnitude Distribution and the Ratio between the Numbers of Long- and Short-Period Comets". Icarus, 73, 149-162.

Jewitt, D.C. and Meech, K. : 1988, "Optical properties of cometary nuclei and a preliminary comparison with asteroids". Astrophys. J., 328, 974-986.

Keller, H.U. : 1989, "Comets - dirty snowballs or icy dirtballs?". In Physics and Mechanics of Cometary Materials, ESA SP-302 (J. Hunt, T.D. Guyenne, Eds.), 39-45, ESA Publ. Div.

Keller, H.U. : 1990, "The Nucleus". In Physics and Chemistry of Comets (W.F. Huebner, Ed.), 13-68, Springer-Verlag.

Kitamura, Y.: 1986, "Axisymmetric Dusty Gas Jet in the Inner Coma of a Comet". Icarus, 66, 241-257.

Klinger, J. : 1980, "Influence of a Phase Transition of Ice on the Heat and Mass Balance of Comets". Science, 209, 271-272.

Klinger, J. : 1983, "Classification of Cometary Orbits Based on the Concept of Orbital Mean Temperature". Icarus, 55, 169-176.

Kochan, H., Roessler, K., Ratke, L., Heyl, M., Hellmann, H. and Schwehm, G. : 1989, "Crustal strength of different model comet materials". In Physics and Mechanics of Cometary Materials, ESA SP-302 (J. Hunt, T.D. Guyenne, Eds.), 115-119, ESA Publ. Div.

Kouchi, A., Greenberg, J.M., Yamamoto, T. and Mukai, T. : 1992, "Extremely low thermal conductivity of amorphous ice : relevance to comet evolution". Astrophys. J. Lett., 388, L73-L76.

Kührt, E. and Keller, H.U. : 1993, "The formation of cometary surface crusts". Icarus, in press.

Langevin, Y., Kissel, J., Bertaux, J.-L. and Chassefière, E. : 1987, "First statistical analysis of 5000 mass spectra of cometary grains obtained by PUMA 1 (Vega 1) and PIA (Giotto) impact ionization mass spectrometers in the compressed modes". Astron. Astrophys., 187, 761-766.

Lunine, J., Engel, S., Rizk, B. and Horányi, M. : 1991, "Sublimation and Reformation of 
Icy Grains in the Primitive Solar Nebula". Icarus, 94, 333-344.

McDonnell, J.A.M., McBride, N., Beard, R., Bussoletti, E., Colangeli, L., Eberhardt, P., Firth, J.G., Grard, R., Green, S.F., Greenberg, J.M., Grün, E., Hughes, D.W., Keller, H.U., Kissel, J., Lindblad, B.A., Mandeville, J.-C., Perry, C.H., Rembor, K., Rickman, H., Schwehm, G., Turner, R.F., Wallis, M.K. and Zarnecki, J.C. : 1993, "Dust Impact Detection System : first results from the Giotto Extended Mission encounter with Comet Grigg-Skjellerup". Nature, 362, 732-734.

Meech, K.J. : 1993, "Observations of the cessation of activity in Comets P/Halley, Torres $(1987 \mathrm{~V})$, and Bowell (1982 I) and implications for the mechanism of the P/Halley outburst". In Workshop on the Activity of Distant Comets (W.F. Huebner, H.U. Keller, D. Jewitt, J. Klinger, R. West, Eds.), 12-20, Southwest Research Institute.

Mumma, M.J., Weissman, P.R. and Stern, S.A.: 1993, "Comets and the Origin of the Solar System : Reading the Rosetta Stone". In Protostars and Planets III (E.H. Levy, J.I. Lunine, M.S. Matthews, Eds.), 1177-1252, Univ. of Arizona Press, Tucson.

Pätzold, M., Edenhofer, P., Bird, M.K. and Volland, H. : 1993, "The Giotto encounter with comet P/Grigg-Skjellerup : first results from the Giotto Radio-Science Experiment". Astron. Astrophys. Lett., 268, L13-L16.

Peale, S.J. : 1990, "On the Density of Halley's Comet". Icarus, 82, 36-49.

Prialnik, D. and Bar-Nun, A. : 1987, "On the evolution and activity of cometary nuclei". Astrophys. J., 313, 893-905.

Prialnik, D. and Bar-Nun, A. : 1988, "The Formation of a Permanent Dust Mantle and Its Effect on Cometary Activity". Icarus, 74, 272-283.

Prialnik, D. and Bar-Nun, A. : 1993, "Distant Outbursts of Comet P/Halley Caused by Crystallization of Amorphous Ice". In Workshop on the Activity of Distant Comets (W.F. Huebner, H.U. Keller, D. Jewitt, J. Klinger, R. West, Eds.), 100-109, Southwest Research Institute.

Prialnik, D., Egozi, U., Bar-Nun, A., Podolak, M. and Greenzweig, Y. : 1993, "On Pore Size and Fracture in Gas-Laden Comet Nuclei". Icarus, in press.

Rickman, H. : 1989, "The nucleus of Comet Halley : surface structure, mean density, gas and dust production". Adv. Space Res., 9, (3)59-(3)71.

Rickman, H. : 1992a, "Cometary nuclei - recent gas flux modelling and applications". Ann. Geophysicae, 10, 157-168.

Rickman, H.: 1992b, "Physico-Dynamical Evolution of Aging Comets". In Interrelations between Physics and Dynamics for Minor Bodies in the Solar System, Goutelas 1991 (D. Benest, C. Froeschlé, Eds.), 197-263, Ed. Frontières.

Rickman, H. and Fernández, J.A. : 1986, "Formation and Blowoff of a Cometary Dust Mantle". In Comet Nucleus Sample Return Mission, ESA SP-249 (O. Melita, Ed.), 185-194, ESA Publ. Div.

Rickman, H., Fernández, J.A. and Gustafson, B.A.S. : 1990, "Formation of stable dust mantles on short-period comet nuclei". Astron. Astrophys., 237, 524-535.

Rickman, H., Festou, M.C., Tancredi, G. and Kamél, L. : 1992, "Twentieth century light curves and the nucleus of comet P/Tempel 2". In Asteroids, Comets, Meteors 1991 (A.W. Harris, E. Bowell, Eds.), 509-512, Lunar and Planetary Institute, Houston.

Sagdeev, R.Z., Elyasberg, P.E. and Moroz, V.I. : 1988, "Is the nucleus of Comet Halley a low-density body?". Nature, 331, 240-242.

Schmitt, B., Espinasse, S., Grim, R.J.A., Greenberg, J.M. and Klinger, J. : 1989, "Laboratory studies of cometary ice analogues". In Physics and Mechanics of Cometary Materials, ESA SP-302 (J. Hunt, T.D. Guyenne, Eds.), 65-69, ESA Publ. Div.

Schmitt, B., Espinasse, S. and Klinger, J. : 1993, "A possible explanation of the outburst of Comet P/Halley at 14 astronomical units from the Sun". In Workshop on the Activity of Distant Comets (W.F. Huebner, H.U. Keller, D. Jewitt, J. Klinger, R. West, Eds.), 124-132, Southwest Research Institute.

Sekanina, Z. : 1982, "The problem of split comets in review". In Comets (L.L. Wilkening, Ed.), 251-287, Univ. Arizona Press. 
Sekanina, Z. : 1984, "Precession model for the nucleus of periodic comet Kopff". Astron. J., 89, 1573-1586.

Sekanina, Z. : 1987, "Nucleus of Comet Halley as a Torque-Free Rigid Rotator". Nature, 325, 326-328.

Sekanina, Z. : 1992, "Effect of discrete-source outgassing on motions of periodic comets and discontinuous orbital anomalies". Astron. J., 105, 702-735.

Shul'man, L.M. : 1972, "The evolution of cometary nuclei". In The Motion, Evolution of Orbits, and Origin of Comets (G.A. Chebotarev, E.I. Kazimirchak-Polonskaya, B.G. Marsden, Eds.), 271-276, D. Reidel Publ. Co.

Shul'man, L.M. : 1981, "A two-layer model of the cometary nucleus". Astrometria i Astrofisica, 45, 21-34.

Shul'man, L.M. : 1992, "On the Intrinsic Source of Energy in Cometary Nuclei : Amorphous Ice or Hydrated Ions?". Bull. Amer. Astron. Soc., 24, 1012 (Äbstract).

Tancredi, G., Rickman, H. and Greenberg, J.M. : 1993, "Thermochemistry of cometary nuclei. I. The Jupiter family case". Astron. Astrophys., in press.

Whipple, F.L. : 1950, "A comet model. I. The acceleration of Comet Encke". Astrophys. J., 111, 375-394.

Whipple, F.L. and Sekanina, Z. : 1979, "Comet Encke : Precession of the spin axis, nongravitational motion and sublimation". Astron. J., 84, 1894-1909.

Whipple, F.L. and Stefanik, R.P.: 1966, "On the physics and splitting of cometary nuclei". Mém. Soc. Roy. Sci. Liège, Sér. 5, 12, 33-52.

Yeomans, D.K. and Chodas, P.C. : 1989, "An asymmetric outgassing model for cometary nongravitational accelerations". Astron. J., 88, 1083-1093. 